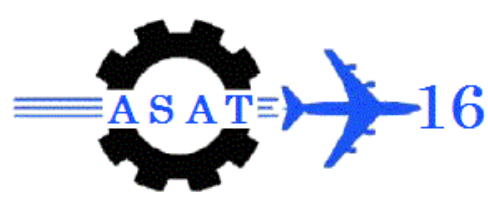

\title{
Advanced Fast Disturbance Rejection PI Controller for DC Motor Position Control
}

\author{
BY \\ Bahaa El-den Gamal ${ }^{*}$, A. N. Ouda , Y. Z. El-Halwagy,+ G. A. El-Nashar†
}

Abstract: This paper presents an approach to an Advanced Fast Disturbance Rejection Proportional-Integral (AFDR-PI) control system based on a benchmark plant model description. The controller is designed for a balance between performance (response time) and robustness (stability margins). The proposed algorithm associated with the mathematical model has been carried out under MATLAB/SIMULINK environment. A comparison between the Proportional-Integral (PI) controller and AFDR-PI controller has been introduced with applied step input disturbance to the input of the control system. The conclusion is that the AFDR-PI controller has the same tracking performance (time response requirements) as the PI controller, and succeeds to reject the disturbance signal higher than the PI controller (robustness requirements).

Keywords: Proportional-Integral (PI) controller, DC motor position control, AFDR-PI controller, Load disturbance rejection

\section{Introduction}

Because of their high reliabilities, flexibility and low cost, DC motors are widely used in industrial application, robot manipulators and home appliances where speed and position control of motor are required. Two main problems encountered in motor control are the timevarying nature of motor parameters under operating conditions and existence of noise in system loop. Analysis and control of complex, nonlinear and/or time-varying systems is a challenging task using conventional methods because of uncertainties [1].

Proportional integral (PI) controllers are commonly used for motor control application because of their simple structures, fast and solid, as nowadays they still represent the most popular choice in most industrial process control applications.

The main reasons for their success are reduced number of parameters, well-established tuning rules, and good performances. The most popular tuning methods were given by Ziegler and Nichols (1942) [8], Astrom and Hagglund (1984), and more recently Zhuang and Atherton (1993) and Luyben and Eskinat (1994) [10]. Different tuning methods are due to different control objectives (e.g. reference following, disturbance rejection) and different plants (e.g. first-order model, second-order model) [2]. 
The PI controller calculation involves two separate parameters: proportional and integral values. The proportional value determines the reaction of the current error. The integral value determines the reaction based on the sum of recent errors. The weighted sum of these two actions is used to adjust the process via the final control element $[3,4]$. For some application, observation the closed-loop system response to disturbance at the plant input is very important to adjustment controller design parameters. In many situations proportional integral (PI) controller cannot achieve robustness requirements, so to make a PI controller perform well for both reference tracking and disturbance rejection is to upgrade it to an AFDR-PI controller. AFDR-PI controller an extended to PI controller, it improves reference tracking response by providing an additional tuning parameters (b) that allows independent control of the impact of the reference signal on the proportional action. AFDR-PI controller succeeds to reject the disturbance signal without further tuning of the parameter. Then the parameters may be slightly varied to obtain the user-defined performance of the real-time control system [4].

This paper is organized as follows: In the next section, fairly description to problem and design control requirements. In Section 3, the system identification toolbox used to obtain a transfer function between shaft position and applied armature voltage for a dc motor by applying plant identification technique built in MATLAB/SIMULINK environment. In Section 4, analysis, design and tuning of proportional-integral (PI) controller are reviewed and a crisp PI control system is designed in MATLAB with the proposed design procedure. In Section 4, observation the closed-loop system response to disturbance at the plant input and modification controller designs to achieve the load disturbance rejection requirements. In Section 5, An AFDR-PI controller obtained in order to design a controller achieves specific requirements for fast disturbance rejection performance with time response requirements. In Section 6, the simulation results with a comparison between the (PI) controller and AFDR-PI controller has been shown. In the last section final conclusions are given and future work is outlined.

\section{Problem Formulation}

A typical problem with the design of a feedback controller is to achieve at the same time a high performance both in the set-point following and in the load disturbance rejection performance. Roughly speaking, a fast load disturbance rejection is achieved with a high-gain controller, which gives an oscillatory set-point step response on the other side [10].

The design controller is used for a wide range of problems: process control, motor drives, automotive, flight control, instrumentation, etc [5]. Such as in many situations, Atmospheric turbulence is one of important factors to affect UAV, missile, and Autonomous Aerial vehicles flight smoothly [6].

The proposed work is position control with specific requirements for disturbance rejection performance of a Permanent Magnet DC (PMDC) motor platform by using modified classical controller design and software implementation in MATLAB/SIMULINK environment. AFDR-PI controller is a suitable solution to reduce the influence of disturbance without changes the PID controller gains.

The main objective of this research is to identify the way to analysis and select a controller type according to closed loop time response requirements to improve the natural response of the overall system, and design the controller to achieve the design requirements with specific requirements for disturbance rejection performance. Implementation of the proposed work is done by software implementation.

The design requirements are for the closed loop system to track a reference input with a rise time less than 1s, settling time less than $2 \mathrm{~s}$, Maximum overshoot $\leq 10 \%$, and zero steady-state error with settling time input disturbance rejection $\leq 1 \mathrm{~s}[1]$. 


\section{Plant Identification}

In many situations, a dynamic representation of the system you want to control is not readily available. One solution to this problem is to obtain a dynamical model using identification techniques. The system is excited by a measurable signal and the corresponding response of the system is collected at some sample rate. The resulting input-output data is then used to obtain a model of the system such as a transfer function or a state-space model.

The dynamical behavior of many systems can be described adequately by a linear relationship between the system's input and output. By experiment that excites the system only in its linear range of behavior and collect the input/output data. Then use the data to estimate a plant model, and design a proposed controller for the obtained model [7].

In modeling the DC motor, the aim is to find the governing differential equations that express the motor characteristics and relate the applied voltage to shaft position. A diagram of the experimental setup is given in figure (1).

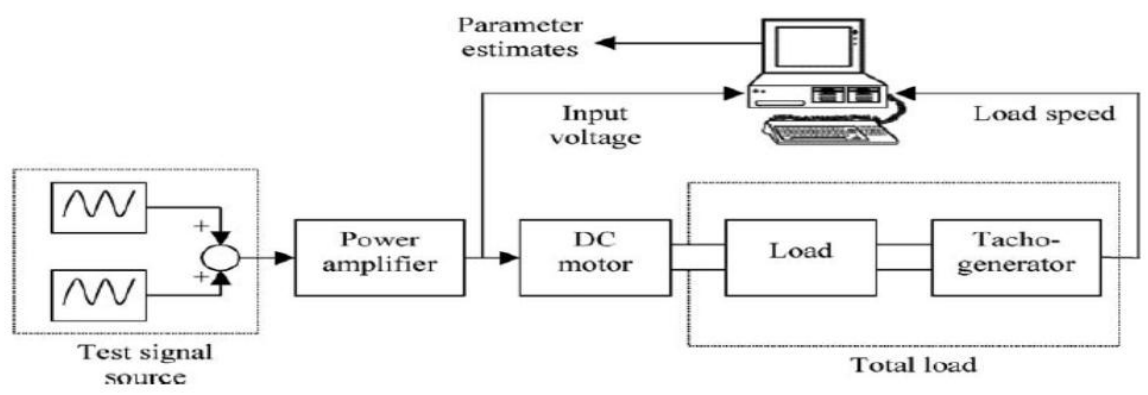

Fig. (1) A diagram of the experimental setup
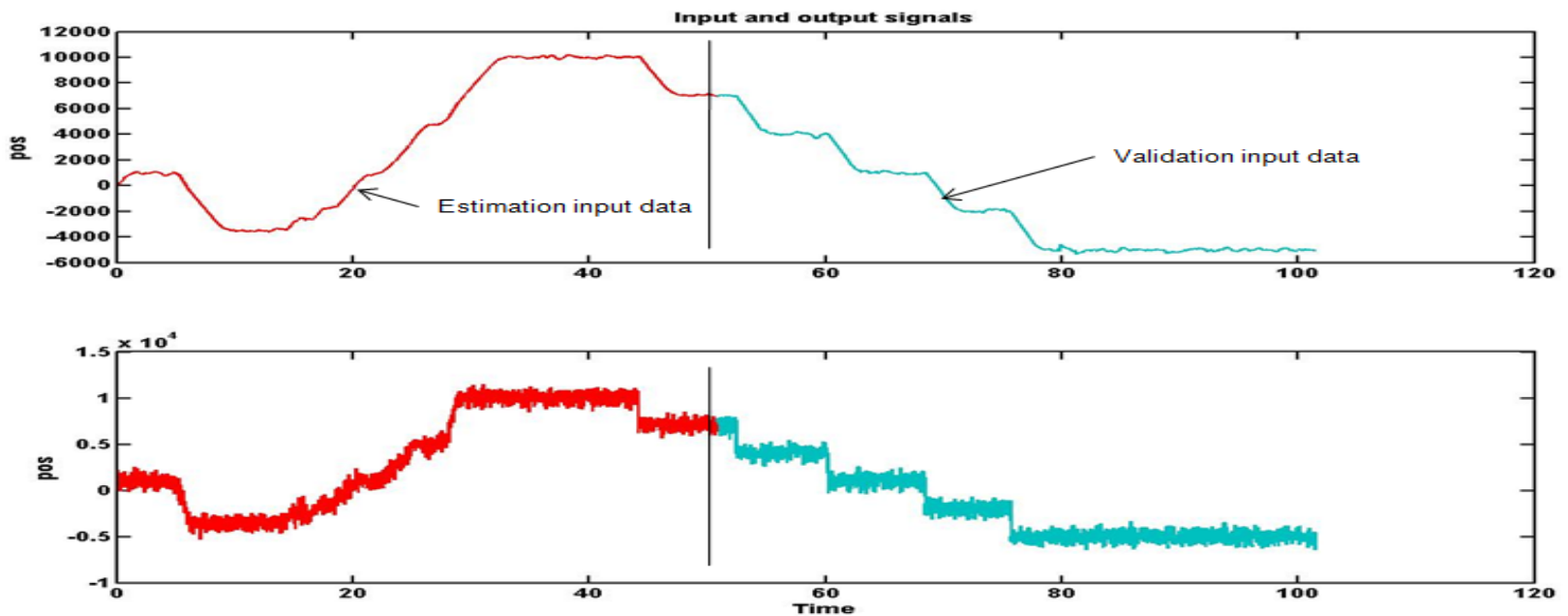

Fig. (2) The test signal (armature voltage)

Two signal generators are used to provide the test signal to the motor. The generators produce sinusoids of different amplitudes and frequencies, which are summed to constitute a persistently exciting signal for the identification process [7]. The signals are carefully adjusted to provide very low speed operation, which is essential for examination of the system nonlinearities. The measured input-output data are collected in a computer using a data acquisition card. The data acquisition card permits use of user defined programs interfaced with MATLAB. The output speed is obtained from the tachometer. The output shaft speed is also measured from an optical sensor as rev/s that is connected to the motor shaft. In a DC motor without or with a rate feedback, the speed of the control motor is proportional to shaft position [3]. The test signal (armature voltage) and the response of the system are given in figure (2). And the fitting percentages of the obtained model are given in figure (3). 


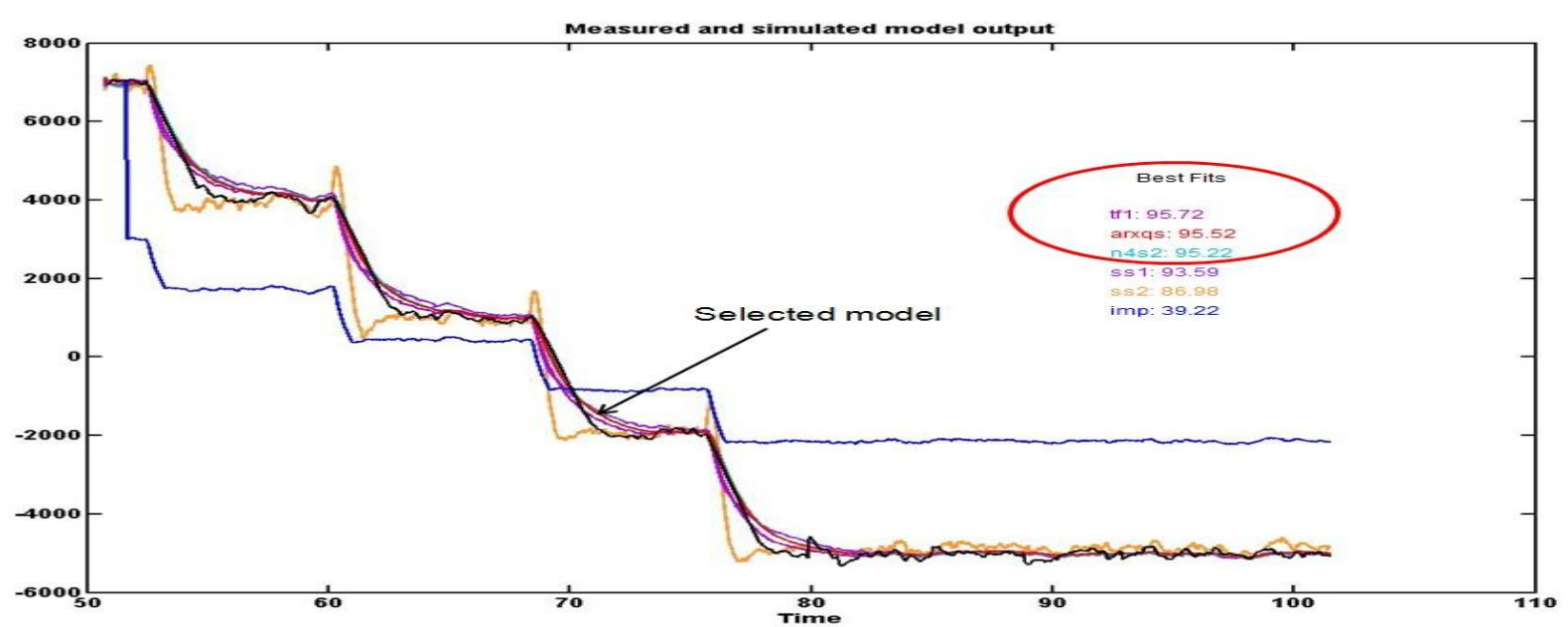

Fig. (3) Simulated model output

Then the obtain transfer function between shaft position and armature voltage at no-load is:

$$
\frac{\theta(s)}{V(s)}=e^{-0.04 s} * \frac{9695}{\mathrm{~s}^{2}+1.28 e 04 s+9632}
$$

\section{Conventional Controller Design}

The selection of the PID parameters is obviously the crucial issue in the overall controller design. This operation should be performed in accordance to the control specifications. Usually, as already mentioned, they are related either to the set-point following or to the load disturbance rejection task, but in some cases both of them are of primary importance. The combination of the proportional, integral, and derivative actions can be done in different ways. In the so-called ideal or non-interacting form, the PID controller is described by the following transfer function [10]:

$$
\begin{gathered}
C(s)=K_{p}\left(1+\frac{1}{T_{i} S}+T_{d} S\right) \\
u(s)=K_{p}\left[r(s)-y(s)+\frac{1}{s T_{i}}\left(r(s)-y(s)+\frac{s T_{d}}{1+\frac{s T_{d}}{N}}(r(s)-y(s))\right]\right.
\end{gathered}
$$

Where $\mathrm{r}(\mathrm{s}), \mathrm{y}(\mathrm{s})$ and $\mathrm{u}(\mathrm{s})$ are the Laplace transforms of the reference, process output and control signal respectively. $K_{p}$ is the where $K_{p}$ is the proportional gain, $K_{p} / T_{i}$ is the integral gain (sometimes denoted as $\mathrm{K}_{\mathrm{i}}$ ), $\mathrm{K}_{\mathrm{p}} \mathrm{T}_{\mathrm{d}}$ is the derivative gain (sometimes denoted as $\mathrm{K}_{\mathrm{d}}$ ), and finally $\mathrm{N}$ is the ratio between $\mathrm{T}_{\mathrm{d}}$ and the time constant of an additional pole introduced to assure the properness of the controller.

PID controllers are usually tuned using hand-tuning or Ziegler-Nichols methods [8]. The general effects of control parameters are summarized in Table (1). The Control System Toolbox offers a variety of functions that allow us to examine the system's characteristics. The step response of the motor model is shown in figure (4).

Table (1) Effect of PID gains on the response

\begin{tabular}{c|c|c|c}
\hline \hline Operation & Rise Time & Overshoot & Stability \\
\hline $\mathrm{K}_{\mathrm{p}}$ & Faster & Increases & Decreases \\
\hline $\mathrm{K}_{\mathrm{i}}$ & Slower & Decreases & Increases \\
\hline $\mathrm{K}_{\mathrm{d}}$ & Faster & Increases & Decreases \\
\hline \hline
\end{tabular}


Considering a closed loop system shown in figure (5), and using the PID Tuner in MATLAB to design a SISO PID controller, the effect of different type of designed controllers, which achieve the design requirements [9], is shown in figure (6).

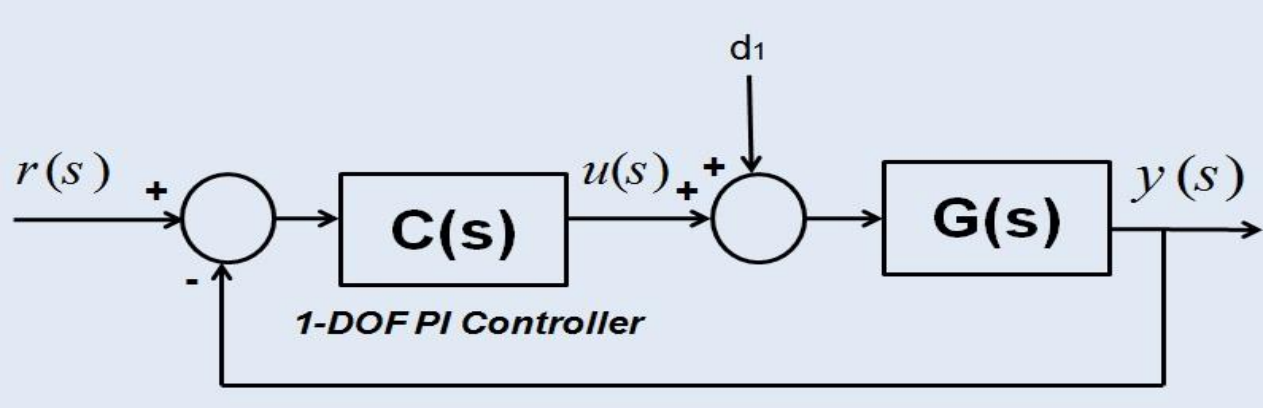

Fig. (4) The system's characteristic

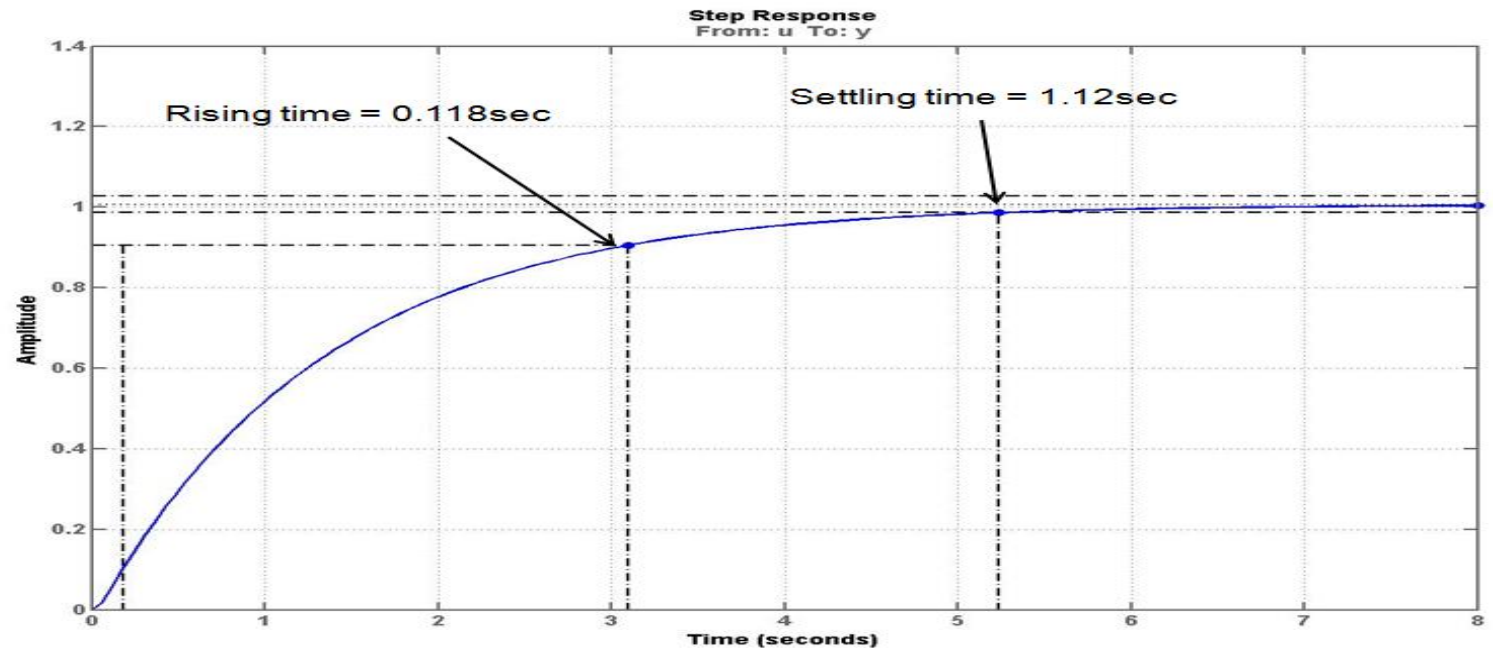

Fig. (5) 1-DOF PI feedback control system.

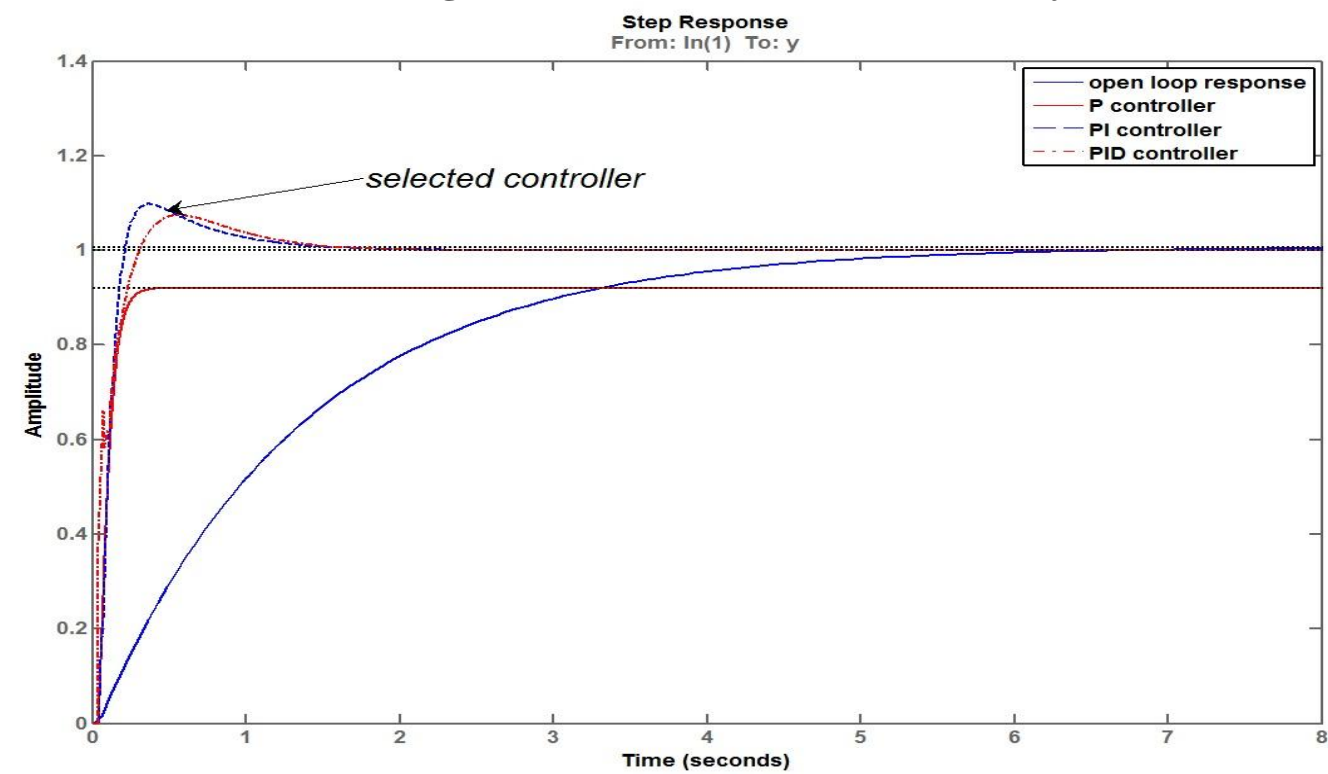

Fig. (6) The system's characteristic with different type of PID controllers.

A PI controller was designed to control the DC motor according to open loop response of the plant, which achieves the time response design requirement as shown in figure (6). Figure (7) shows output and input disturbance rejection signals of PI control system with adjusted parameters. For step input, the settling time is about 1.12 seconds, the overshoot is about 9.81 
percent, and zero steady-state error which is acceptable for design requirement when the controller parameters are $\mathrm{K}_{\mathrm{p}}$ gain is 11.45 , and $\mathrm{K}_{\mathrm{i}}$ is 22.53. Then assume that a step disturbance occurs at the plant input and the main purpose of the PI controller is to reject this disturbance quickly. The peak deviation is about 0.4 and it settles to less than 0.1 in about 1.95 seconds. Because the attenuation of low frequency disturbance is inversely proportional to integral gain $\mathrm{K}_{\mathrm{i}}$, maximizing the integral gain is a useful heuristic to obtain a PI controller with fast disturbance rejection [4].Using PID tuner in MATLAB/SIMULINK, the $\mathrm{K}_{\mathrm{i}}$ gain in the Controller parameters increases and then decreases, with the maximum value occurring at 36.42. When $\mathrm{K}_{\mathrm{i}}$ is 36.42 , the peak deviation is reduced to 0.07 (about $17.5 \%$ improvements) and it settles to less than 0.1 in about 0.962 seconds (about $13.3 \%$ improvement) [4] as shown in figure (8).
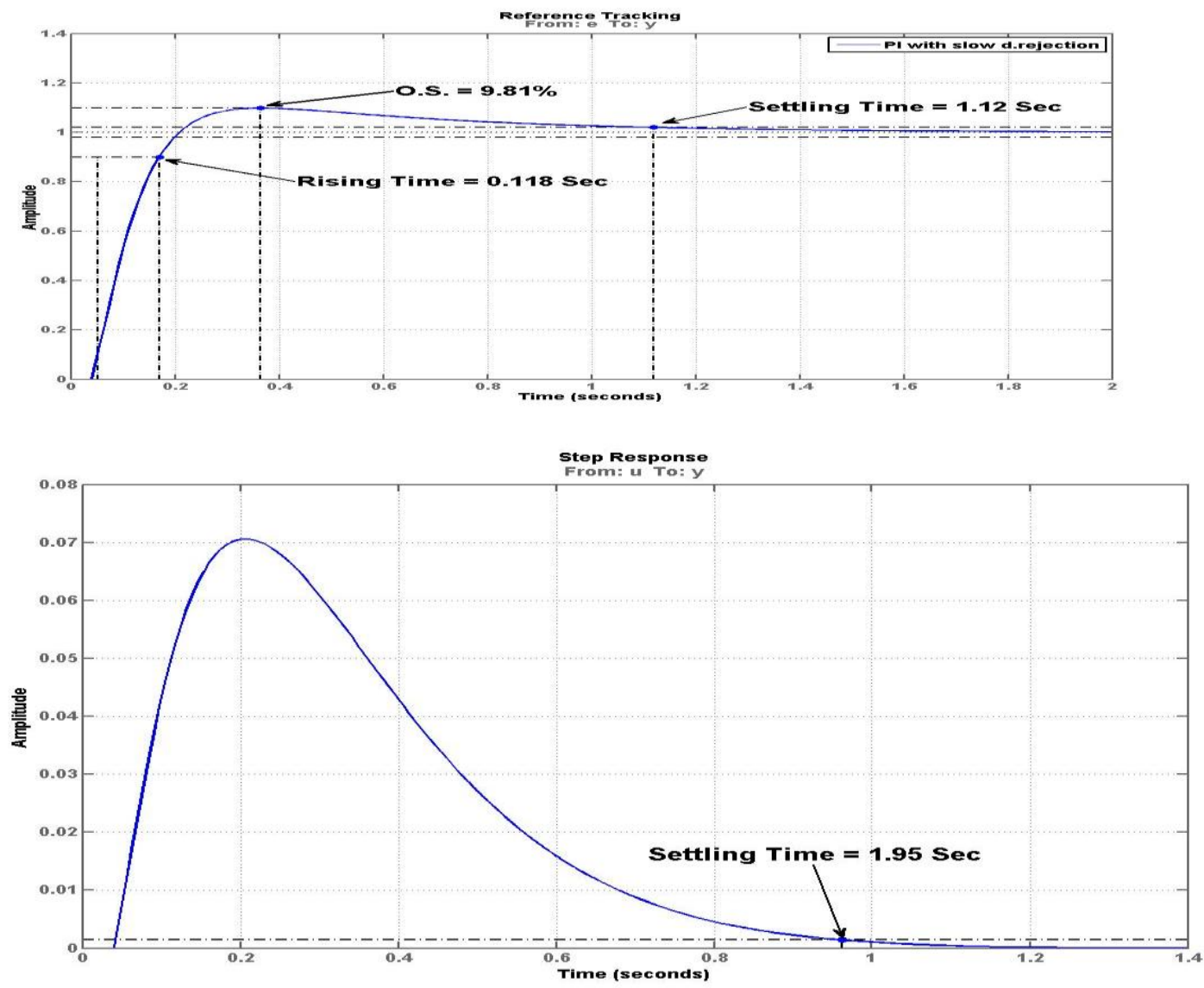

Fig. (7) Output and input disturbance rejection signals of PI control system 

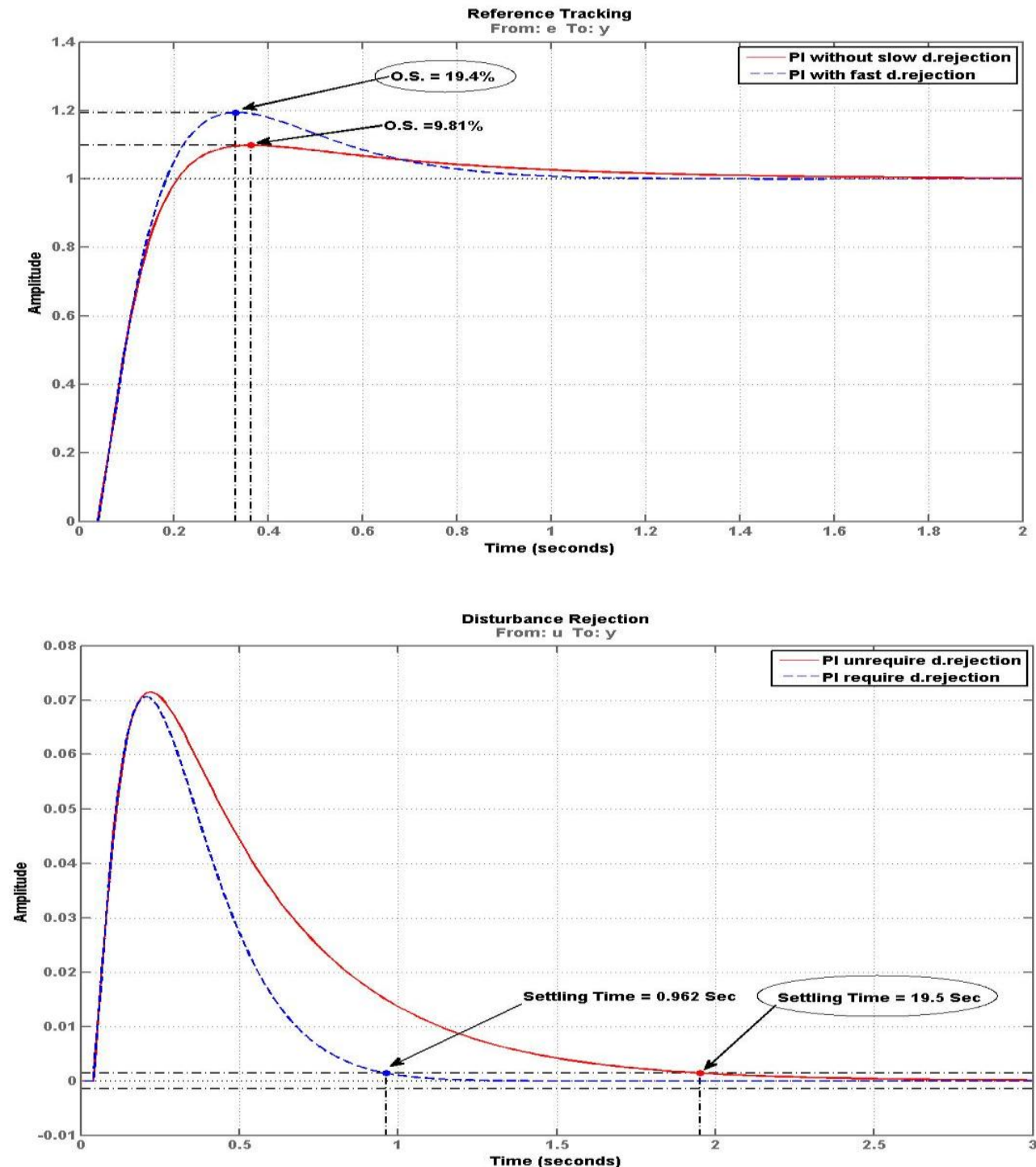

Fig. (8) Output and input disturbance rejection signals of PI control system with fast disturbance rejection

Because increasing the bandwidth, the step reference tracking response becomes more oscillatory. Additionally the overshoot exceeds 10 percent, which is usually unacceptable. This type of performance tradeoff between reference tracking and disturbance rejection often exists because a single PI controller is not able to satisfy both design goals at the same time [4]. The comparison between effects of control parameters are summarized in Table (2).

Table (2) Comparison between effects of control parameters

\begin{tabular}{c|c|c}
\hline \hline $\begin{array}{c}\text { Controller } \\
\text { parameters }\end{array}$ & $\begin{array}{c}\text { PI controller without achieve } \\
\text { robustness requirements }\end{array}$ & $\begin{array}{c}\text { PI controller with achieve } \\
\text { robustness requirements }\end{array}$ \\
\hline $\mathrm{K}_{\mathrm{p}}$ & 11.45 & 10.99 \\
\hline $\mathrm{K}_{\mathrm{i}}$ & 22.53 & 36.42 \\
\hline Rise Time & 0.118 second & 0.108 second \\
\hline Settling Time & 1.12 second & $19.4 \%$ \\
\hline Overshoot & $9.81 \%$ & stable \\
\hline $\begin{array}{c}\text { Closed-loop } \\
\text { stability }\end{array}$ & stable & \\
\hline \hline
\end{tabular}




\section{Disturbance Rejection Tuning}

The equation (3) of a PID controller given in the previous section are actually not adopted in practical cases because of a few problems that can be solved with suitable modifications of the basic control law [2]. These are analyzed in this section. This problem can be approached by designing a two-degree-of-freedom control architecture, namely, a combined feed forward/feedback control law [10]. It improves reference tracking response by providing additional tuning parameters (b) that allows independent control of the impact of the reference signal on the proportional action. In figure (9) AFDR-PI structure is shown, there is a feedback controller $\mathrm{C}$ and a feed-forward filter $\mathrm{F}$ [4].The AFDR-PI control law [9, 11, 12] can be obtained as:

$$
u(s)=K_{p}\left[b r(s)-y(s)+\frac{1}{s T_{i}}\left(r(s)-y(s)+\frac{s T_{d}}{1+\frac{s T_{d}}{N}}(c r(s)-y(s))\right]\right.
$$

In case of AFDR-PI controller, $T_{d}=0$

$$
u(s)=r(s)\left[b K_{p}+\frac{K_{i}}{s}\right]-y(s)\left[K_{p}+\frac{K_{i}}{s}\right]
$$

Where $\mathrm{r}(\mathrm{s}), \mathrm{y}(\mathrm{s})$ and $\mathrm{u}(\mathrm{s})$ are the Laplace transforms of the reference, process output and control signal respectively. $K_{p}$ is the where $K_{p}$ is the proportional gain, $K_{p} / T_{i}$ is the integral gain (sometimes denoted as $K_{i}$ ) and $K_{p} T_{d}$ is the derivative gain (sometimes denoted as $K_{d}$ )., and finally $\mathrm{N}$ is the ratio between $\mathrm{T}_{\mathrm{d}}$ and the time constant of an additional pole introduced to assure the properness of the controller. Parameters $b$ and $c$ are called set-point weights and constitute a simple way to obtain a 2 - DOF controller as shown in figure $(10)[8,11,12]$.

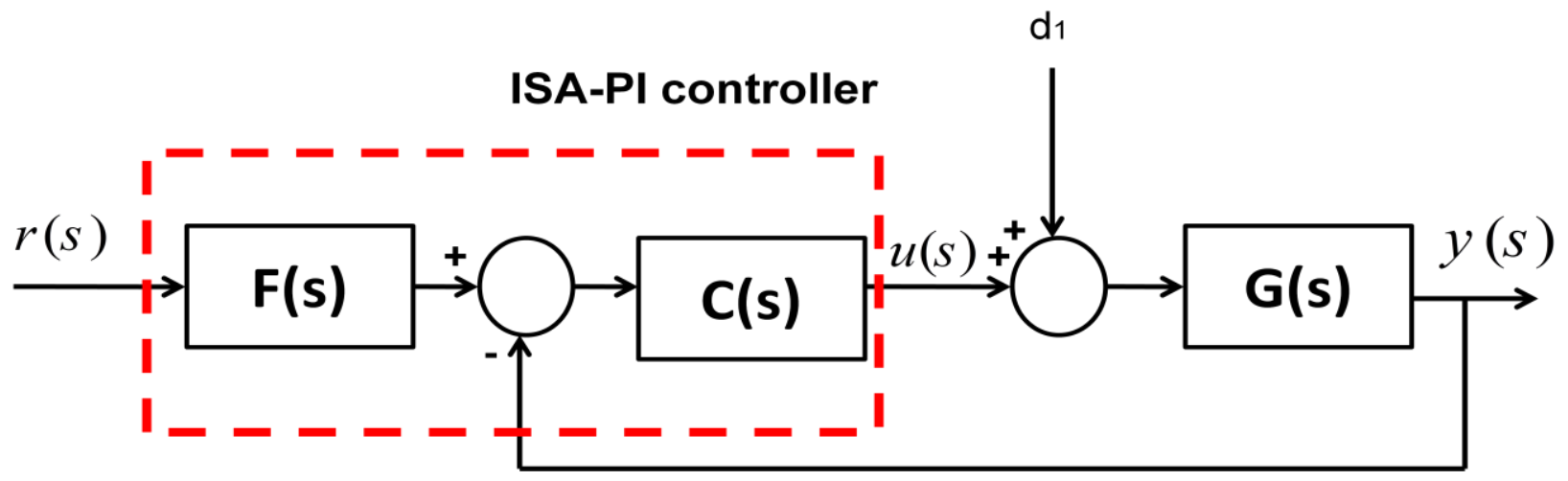

Fig. (9) AFDR-PI structures

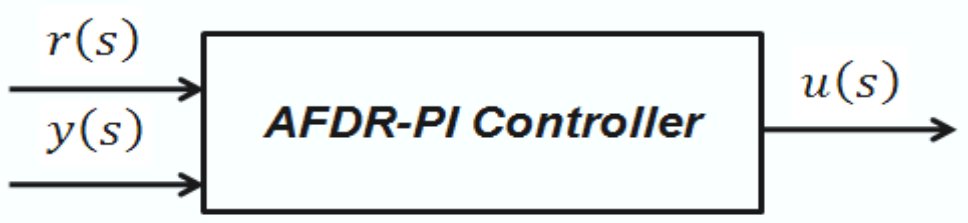

Fig. (10) 2- DOF AFDR-PI controller

In this design, $\mathrm{C}$ is a regular PI controller in parallel form that can be represented by a PI object:

$$
C(s)=\operatorname{pid}\left(K_{p}, K_{i}\right)=K_{p}+\frac{K_{i}}{s}
$$


$\mathrm{F}$ is a pre-filter that involves $\mathrm{K}_{\mathrm{p}}$ and $\mathrm{K}_{\mathrm{i}}$ gains from $\mathrm{C}$ plus the set point weight (b):

$$
F(s)=\frac{b K_{P} s+K_{i}}{K_{p} s+K_{i}}=\frac{b *\left(s+\frac{K_{i}}{K_{p}}\right)}{\left(s+\frac{K_{i}}{K_{p}}\right)}
$$

Therefore the AFDR-PI controller has two inputs ( $\mathrm{r}$ and $\mathrm{y}$ ) and one output (u).Set-point weight (b) is a real number between 0 and 1 . When it decreases, the overshoot in the reference tracking response is reduced [4]. Figure (11) shows the function of the set-point weight is to smooth the (step) set-point signal in order to damp the response to a set-point change.

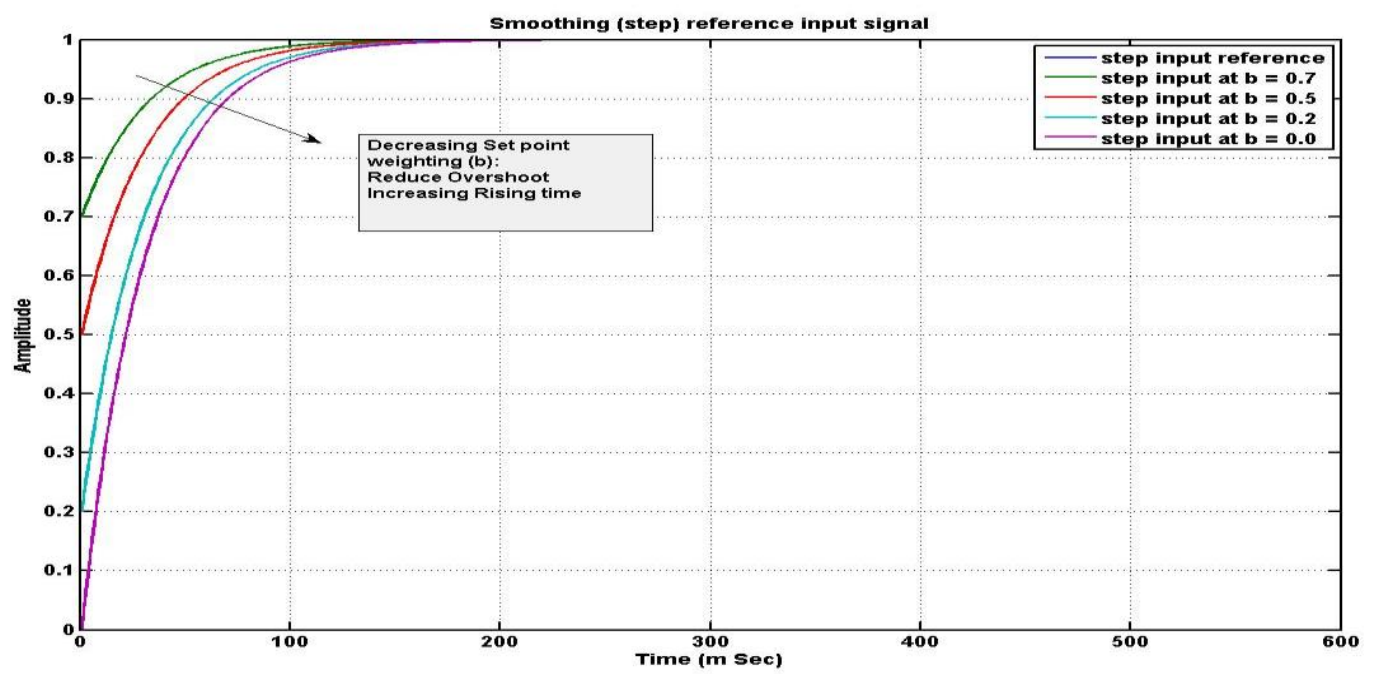

Fig. (11) Smoothing (step) set-point signal

\section{Simulation Result}

It appears that the load disturbance rejection task is decoupled from the set-point following one and obviously it does not depend on the weight (b). Thus, the PI parameters can be selected to achieve a high load disturbance rejection performance and then the set-point following performance can be recovered by suitably selecting the value of the parameter (b) [10]. Figure (12) shows the output signals of AFDR-PI control system with different value of set-point weight (b). Figure (13) shows the output and input disturbance rejection signals of AFDR-PI control system for achieve required time response specification with fast disturbance rejection $(a t b=0.7)$. The reference tracking response with AFDR-PI controller has much less overshoot because set point weight (b) reduces overshoot and The disturbance rejection responses are the same because set-point weight (b) only affects reference tracking [4]. 


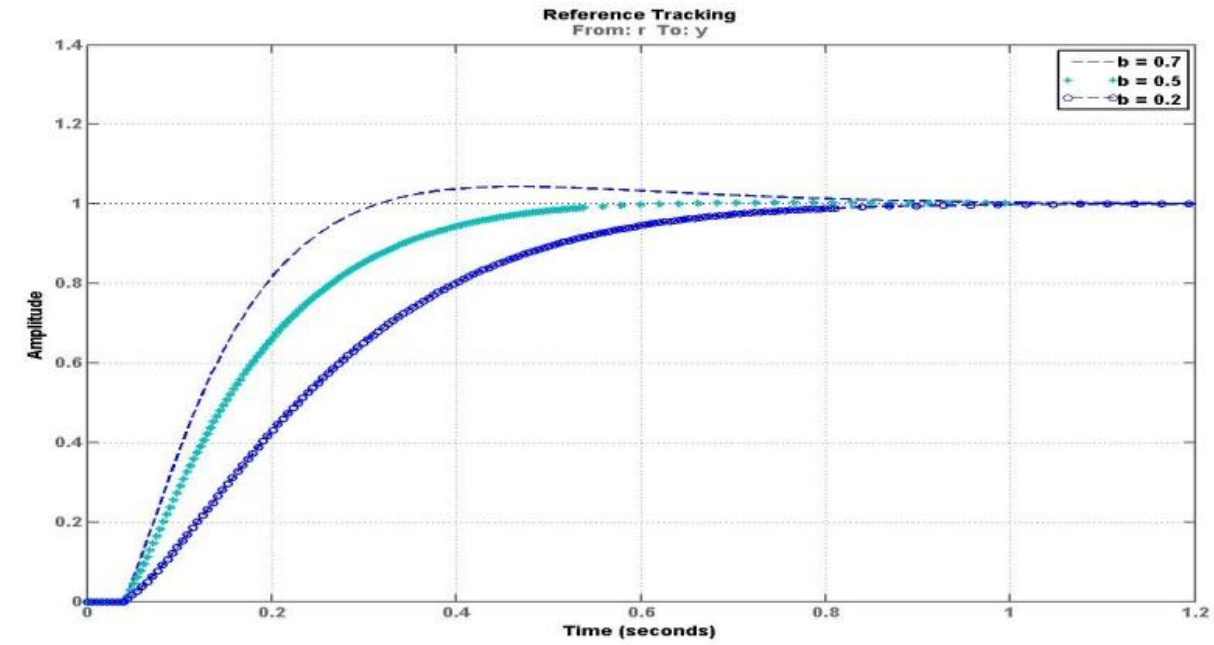

Fig. (12) Output signals of AFDR-PI control system with fast disturbance rejection with different value of set-point weight $b$
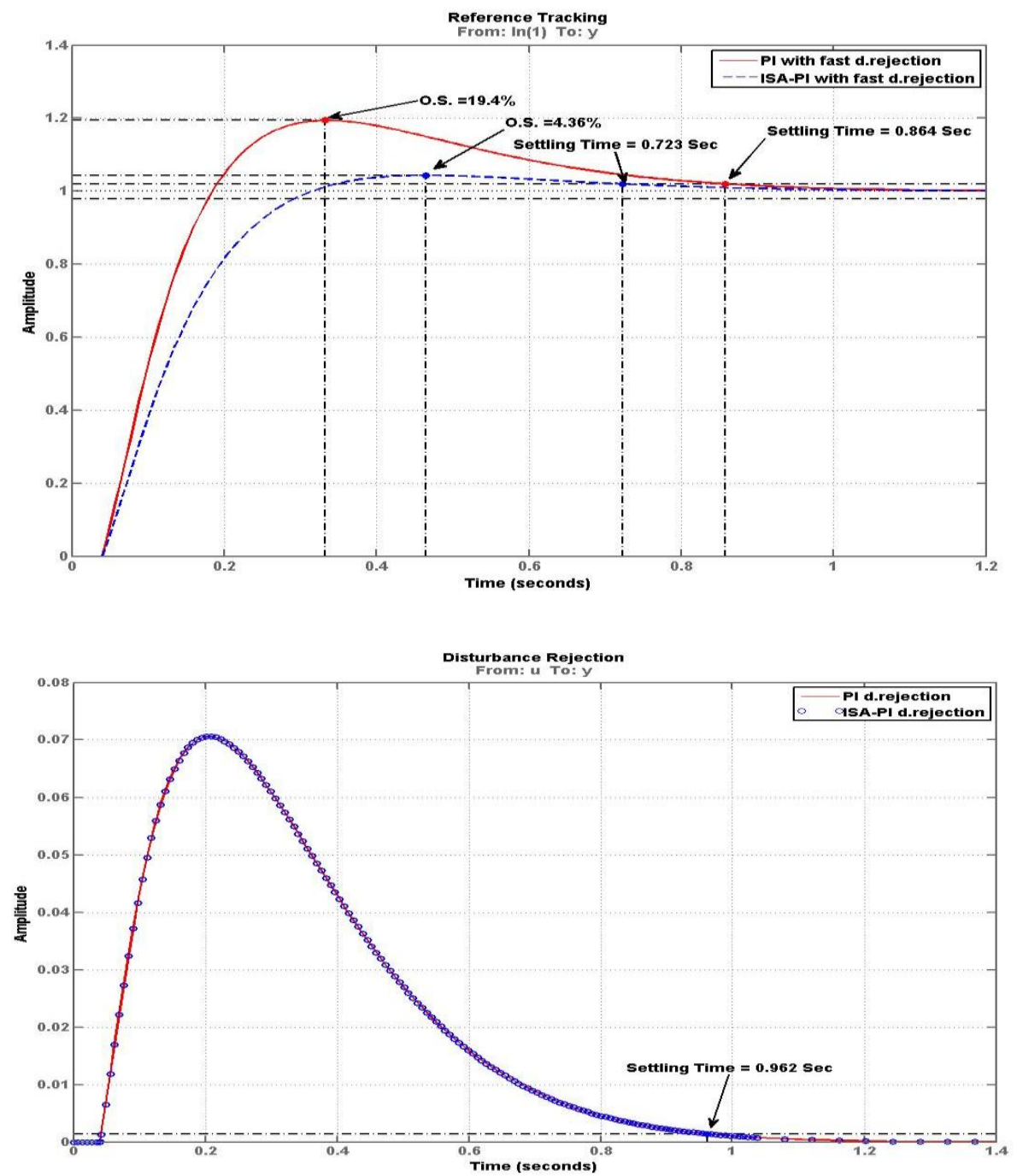

Fig. (13) Output and input disturbance rejection signals of AFDR-PI control system with fast disturbance rejection. 


\section{Conclusions and Future work:}

In this paper, the design process description of an AFDR-PI and PI controllers, and give an analysis of the efficiency of the control model in dynamic characteristics of tracking and eliminating the load disturbance effect. Parameters of PI were tuned using a PID Tuner instead of conventional tuning methods. The final parameter values were adjusted in just a few iterations in order to achieve design requirement. Thus the time and effort for tuning parameters decreased considerably. The performance criteria are defined in time domain where the transient response of the system to a step input was considered.

AFDR-PI controller an extended to PI controller, it improves reference tracking response by providing an additional tuning parameters (b), Set-point weight (b) is a real number between 0 and 1. When it decreases, the overshoot in the reference tracking response is reduced [4].

AFDR-PI and PI controllers are designed, and simulation analysis is carried out finally. The simulation results show that, the tracking performance of AFDR-PI controller is fairly like as traditional PI controller designed in this paper, but the anti-disturbance performance is better than PI controller.

As a future work, modified the proposed controller parameters to be suitable connection with $6 \mathrm{DOF}$ flight simulation model of generic platform, and compare the difference between the classical controller effect and proposed controller. Realization the controller in different embedded systems to compare the effect of the different processor in the control action and compare between the modified classical control techniques and advanced control techniques in hardware in the loop (HWIL) experimental to show the effect of realization controller on the flight simulation.

\section{References:}

[1] ManafeddinNamazov, OnurBasturk, "DC motor position control using fuzzy proportional-derivative controllers with different defuzzification methods", TJFS: Turkish Journal of Fuzzy Systems (eISSN: 1309-1190).

[2] Emanuele Crisostomi and Alberto Landi, "From Basic to Advanced PI Controllers:A Complexity vs. Performance Comparison", Department of Energy and Systems Engineering, University of Pisa, Italy, December 2013.

[3] EhabSafwat, A. M. Kamel, Y.Z. El-Halwagy, "Design and Realization of an Embedded Control Fin Drive", Proceedings of the 7th ICEENG Conference, 25-27 May, 2014.

[4] Mathworks Inc., Simulink ${ }^{\circledR}$ "Control system toolbox ${ }^{\text {TM }}$ Getting Started Guide", 3rd printing, 2014.

[5] htrom K. and Hagglund T., "The future of PID control, Control Engineering Practice", 2001, 9, NO.1163.

[6] Zhengyun, Rent Hong Zhang, Huihe Shao, "Comparison of PID Control and PPI Control”, Conference on Decision and Control, Maui, Hawaii USA, December 2003.

[7] Ljung, L., "System Identification - Theory for the User", 2nd edition, Prentice Hall, Upper Saddle River, N.J., 1999.

[8] Ogata, K.," Modern Control Engineering”, Third Edition, Prentice Hall, 1997. 
[9] Robert Babuska and Stefano Stramigioli, "Matlab and Simulink for Modeling and Control", November 1999, Faculty of Information Technology and Systems, Delft University of Technology.

[10] Antonio Visioli, "Practical PID Control, Department of Electronic and Electrical Engineering”, University of Strathclyde, United Kingdom, Springer-Verlag London Limited 2006.

[11] K. Astrom and T. Hgglund, "PID Controller", Instrument of Society of America, 1995.

[12] P. Cominos and N. Munro, "Pid controllers: recent tuning methods and design to specification,” IEE Proceedings.Part D., vol. 149, pp.46-53, 2002.

[13] R. Vilanova, and O. Arrieta, "PID design for improved disturbance attenuation: min $\max \quad$ Sensitivity matching approach”, IAENG International Journal of Applied Mathematics, 37:1, IJAM_37_1_6. 